\title{
Integrable systems of the intermediate long wave type in $2+1$ dimensions
}

\author{
B. Gormley ${ }^{1}$, E.V. Ferapontov ${ }^{1,2}$, V.S. Novikov ${ }^{1}$, M.V. Pavlov ${ }^{3}$ \\ ${ }^{1}$ Department of Mathematical Sciences, Loughborough University \\ Loughborough, Leicestershire LE11 3TU, United Kingdom \\ ${ }^{2}$ Institute of Mathematics, Ufa Federal Research Centre \\ Russian Academy of Sciences, 112 Chernyshevsky Street \\ Ufa 450008, Russian Federation \\ ${ }^{3}$ Lebedev Physical Institute \\ Russian Academy of Sciences, \\ Leninskij Prospekt 53, 119991 Moscow, Russia \\ e-mails: \\ B.Gormley@lboro.ac.uk \\ E.V.Ferapontov@lboro.ac.uk \\ mpavlov@itp.ac.ru \\ V.Novikov@lboro.ac.uk
}

\begin{abstract}
We classify $2+1$ dimensional integrable systems with nonlocality of the intermediate long wave type. Links to the $2+1$ dimensional waterbag system are established. Dimensional reductions of integrable systems constructed in this paper provide dispersive regularisations of hydrodynamic equations governing propagation of long nonlinear waves in a shear flow with piecewise linear velocity profile (for special values of vorticities).
\end{abstract}

MSC: 35Q55, 37K10.

Keywords: multi-dimensional integrable systems, hydrodynamic reductions, dispersive deformations, Lax pairs, waterbag system.

\section{Introduction}

The starting point of this paper is the $2+1$ dimensional integrable equation

$$
u_{t}=u u_{y}+\frac{\epsilon}{2} \frac{T+1}{T-1} u_{y y},
$$

which first appeared in [4] as a differential-difference $\mathrm{KP}$ equation, see also [19. Here $u=u(x, y, t)$ and the symbol $T$ denotes $\epsilon$-shift in the $x$-variable: $T u(x, y, t)=u(x+\epsilon, y, t)$. Introducing the nonlocal variable $w=\frac{\epsilon}{2} \frac{T+1}{T-1} u_{y}$, known as nonlocality of the intermediate long wave (ILW) type, we can represent equation (11) in the form

$$
u_{t}=u u_{y}+w_{y}, \quad \triangle w=\frac{T+1}{2} u_{y},
$$

where $\triangle=\frac{T-1}{\epsilon}$ is the discrete $x$-derivative. In the dispersionless limit, $\epsilon \rightarrow 0$, we obtain the system $u_{t}=$ $u u_{y}+w_{y}, w_{x}=u_{y}$ which is equivalent to a single PDE, $u_{t}=u u_{y}+\partial_{x}^{-1} u_{y y}$, discussed in [21] as a counterpart of the dispersionless KP equation. 
Generalising example (2), paper [1] gives a classification of $2+1$ dimensional integrable equations of ILW type,

$$
u_{t}=\varphi(u, w) u_{x}+\psi(u, w) u_{y}+\tau(u, w) w_{x}+\eta(u, w) w_{y}+\epsilon(\ldots)+\epsilon^{2}(\ldots), \quad \triangle w=\frac{T+1}{2} u_{y} .
$$

Here dots at $\epsilon$ and $\epsilon^{2}$ denote terms which are homogeneous differential polynomials of degree two and three in the $x$ - and $y$-derivatives of $u$ and $w$, respectively (the coefficients of these polynomials are allowed to be arbitrary functions of $u$ and $w$ ). It turns out that $\epsilon$-terms, as well as all terms containing derivatives with respect to $x$ (in particular, coefficients $\varphi$ and $\tau$ ), must vanish identically, leading to the following classification result.

Theorem 1. [11] The following examples constitute a complete list of integrable equations (3) of ILW type:

$$
\begin{aligned}
& u_{t}=u u_{y}+w_{y}, \\
& u_{t}=\left(w+\alpha e^{u}\right) u_{y}+w_{y}, \\
& u_{t}=u^{2} u_{y}+(u w)_{y}+\frac{\epsilon^{2}}{12} u_{y y y} \\
& u_{t}=u^{2} u_{y}+(u w)_{y}+\frac{\epsilon^{2}}{12}\left(u_{y y}-\frac{3}{4} \frac{u_{y}^{2}}{u}\right)_{y} .
\end{aligned}
$$

In all cases the nonlocality is the same: $\triangle w=\frac{T+1}{2} u_{y}$.

Equation (6) is a differential-difference analogue of the Veselov-Novikov equation discussed in [18. Equation (7) can be viewed as a differential-difference version of the modified Veselov-Novikov equation. Note that although equations (44) and (5) do not contain $\epsilon$-terms, they should still be considered as dispersive due to the form of the nonlocality.

The above classification utilised the perturbative approach of [8, 9] based on the requirement that all hydrodynamic reductions [7] of the dispersionless limit can be deformed into reductions of the full dispersive equation.

\begin{tabular}{|c|c|c|c|}
\hline$E q n$ & Lax pair & $\begin{array}{l}\text { Dispersionless } \\
\text { limit }\end{array}$ & $\begin{array}{l}\text { Dispersionless } \\
\text { Lax pair }\end{array}$ \\
\hline (44) & $\begin{array}{l}T \psi=\epsilon \psi_{y}-u \psi \\
\epsilon \psi_{t}=\frac{\epsilon^{2}}{2} \psi_{y y}+\left(w-\frac{\epsilon}{2} u_{y}\right) \psi\end{array}$ & $\begin{array}{l}u_{t}=u u_{y}+w_{y} \\
w_{x}=u_{y}\end{array}$ & $\begin{array}{l}e^{S_{x}}=S_{y}-u \\
S_{t}=\frac{1}{2} S_{y}^{2}+w\end{array}$ \\
\hline (15) & $\begin{array}{l}T \psi=\epsilon e^{-u} \psi_{y}-\alpha \psi \\
\psi_{t}=\frac{\epsilon}{2} \psi_{y y}+\left(w-\frac{\epsilon}{2} u_{y}\right) \psi_{y}\end{array}$ & $\begin{array}{l}u_{t}=\left(w+\alpha e^{u}\right) u_{y}+w_{y} \\
w_{x}=u_{y}\end{array}$ & $\begin{array}{l}e^{S_{x}}=e^{-u} S_{y}-\alpha \\
S_{t}=\frac{1}{2} S_{y}^{2}+w S_{y}\end{array}$ \\
\hline (6) & $\begin{array}{l}\epsilon(T-1) \psi_{y}=-2 u(T+1) \psi \\
\psi_{t}=\frac{\epsilon^{2}}{12} \psi_{y y y}+\left(w-\frac{\epsilon}{2} u_{y}\right) \psi_{y}\end{array}$ & $\begin{array}{l}u_{t}=u^{2} u_{y}+(u w)_{y} \\
w_{x}=u_{y}\end{array}$ & $\begin{array}{l}\frac{e^{S_{x}}-1}{e^{S_{x}}+1} S_{y}=-2 u \\
S_{t}=\frac{1}{12} S_{y}^{3}+w S_{y}\end{array}$ \\
\hline (17) & $\begin{array}{l}\epsilon(T-1) \psi_{y}=\frac{\epsilon}{2} \frac{u_{y}}{u}(T-1) \psi-2 u(T+1) \psi \\
\psi_{t}=\frac{\epsilon^{2}}{12} \psi_{y y y}+\left(w-\frac{\epsilon}{2} u_{y}\right) \psi_{y}+\frac{1}{2}\left(w_{y}-\frac{\epsilon}{2} u_{y y}\right) \psi\end{array}$ & $\begin{array}{l}u_{t}=u^{2} u_{y}+(u w)_{y} \\
w_{x}=u_{y}\end{array}$ & $\begin{array}{l}\frac{e^{S_{x}}-1}{e^{S_{x}}+1} S_{y}=-2 u \\
S_{t}=\frac{1}{12} S_{y}^{3}+w S_{y}\end{array}$ \\
\hline
\end{tabular}
This method provides an efficient approach to the classification of integrable systems in $2+1$ dimensions.

Lax pairs, dispersionless limits and dispersionless Lax pairs of equations from Theorem 1 are provided in the table below; note that equations (6) and (7) have coinciding dispersionless limits/dispersionless Lax pairs. Here and in what follows, Lax pairs are obtained as quantisations of dispersionless Lax pairs [20. 


\subsection{Summary of the main results}

As a generalisation of ansatz (3), in this paper we classify integrable systems of the form

$$
\begin{aligned}
& u_{t}=\alpha(u, v, w) u_{y}+\beta(u, v, w) v_{y}+\gamma(u, v, w) w_{y}+\epsilon(\ldots) \\
& v_{t}=\phi(u, v, w) u_{y}+\psi(u, v, w) v_{y}+\eta(u, v, w) w_{y}+\epsilon(\ldots) \\
& \triangle w=\frac{T+1}{2} u_{y}
\end{aligned}
$$

Here dots at $\epsilon$ denote homogeneous differential polynomials of degree two in the $y$-derivatives of $u, v$ and $w$, whose coefficients are allowed to be arbitrary functions of $u, v, w$ (one can show that the coefficients at $x$ derivatives have to vanish identically). This classification problem has several important differences from that of Theorem 1. First of all, no cases of Theorem 1 contain $\epsilon$-terms (thus, integrable systems of type (8) constitute an essentially multi-component phenomenon). Secondly, there exist no integrable systems of type (8) without $\epsilon$-terms, that is, no analogues of cases (4), (5) of Theorem 1. In what follows, we will assume that the dispersion relation of the dispersionless limit of system (8) is an irreducible cubic curve (see Section 22).

Theorem 2. Modulo point transformations $v \rightarrow f(u, v)$, rescaling of $u, v$ and $w$, and Galilean transformations, there exist six integrable systems of the form (8):

$$
\begin{aligned}
& u_{t}=(u v)_{y}-\epsilon u_{y y} \\
& v_{t}=u u_{y}+v v_{y}+4 w_{y}+\epsilon v_{y y} \\
& u_{t}=w u_{y}+v_{y}+\frac{\epsilon}{2} u_{y y} \\
& v_{t}=\left(v w+\frac{1}{2} v^{2}\right)_{y}-\frac{\epsilon}{2}\left(v_{y y}-\left(v u_{y}\right)_{y}\right) \\
& u_{t}=w u_{y}+v_{y}+\frac{\epsilon}{4} u_{y y} \\
& v_{t}=\frac{v^{2}}{\cosh ^{2} u} u_{y}+(w+2 v \tanh u) v_{y}+v w_{y}-\frac{\epsilon}{4}\left(v_{y y}-\frac{1}{2} v \tanh u u_{y y}-\frac{v}{2 \cosh ^{2} u} u_{y}^{2}-\frac{1}{2} \tanh u u_{y} v_{y}\right) \\
& u_{t}=u u_{y}+v v_{y}+w_{y}+\frac{\epsilon}{4} v_{y y}, \\
& v_{t}=(u v)_{y}-\frac{\epsilon}{4} u_{y y}, \\
& u_{t}=u u_{y}+v_{y}+w_{y}+\frac{i}{4} \epsilon u_{y y} \\
& v_{t}=2 v u_{y}+u v_{y}-\frac{i}{4} \epsilon\left(v_{y y}+w_{y y}-u_{y}^{2}\right) \\
& u_{t}=\left(v e^{u}+w\right) u_{y}+e^{u} v_{y}+w_{y}+\frac{\epsilon}{4}\left(\frac{v_{y}}{\sqrt{v^{2}+\alpha}}\right)_{y} \\
& v_{t}=e^{u}\left(v^{2}+\alpha\right) u_{y}+\left(e^{u} v+w\right) v_{y}-\frac{\epsilon}{4}\left(\sqrt{v^{2}+\alpha}\left(u_{y y}+u_{y}^{2}\right)-\frac{v_{y}^{2}}{\sqrt{v^{2}+\alpha}}\right)
\end{aligned}
$$

In all cases the nonlocality is the same: $\triangle w=\frac{T+1}{2} u_{y}$.

The proof of Theorem 2 is summarised in Section 2

Dispersionless limits of some of the above systems can be obtained as special cases of the $2+1$ dimensional waterbag system,

$$
u_{t}^{i}=u^{i} u_{y}^{i}+w_{y}, \quad w_{x}=\sum_{k=1}^{N} \frac{1}{\omega_{k}} u_{y}^{k},
$$

$i=1, \ldots, N$, which is discussed in Section 3. We show that the waterbag system possesses integrable dispersive deformations for special values of the parameters $\omega_{k}$, thus providing multi-component generalisations of examples from Theorem 2. These deformations are utilised in Section 4 to construct dispersive regularisation of a system governing propagation of long nonlinear waves in a shear flow with piecewise linear velocity profile (for special values of vorticities $\omega_{k}$ ). The most general multi-component integrable system arising from the $2+1$ dimensional 
waterbag system (where we know the structure of dispersive deformations) corresponds to the case when $\omega_{1}=$ $\cdots=\omega_{n}=1$ and $\omega_{n+1}=\cdots=\omega_{n+m}=-1$. Setting $u^{n+\alpha}=v^{\alpha}$ we obtain dispersionless equations

$$
u_{t}^{i}=u^{i} u_{y}^{i}+w_{y}, \quad v_{t}^{\alpha}=v^{\alpha} v_{y}^{\alpha}+w_{y}, \quad w_{x}=\sum_{k=1}^{n} u_{y}^{k}-\sum_{\alpha=1}^{m} v_{y}^{\alpha} .
$$

This system possesses integrable dispersive deformation

$$
\begin{aligned}
& u_{t}^{i}=u^{i} u_{y}^{i}+w_{y}+\frac{\epsilon}{2}\left(v^{1}+\cdots+v^{m}\right)_{y y}+\frac{\epsilon}{2} D_{j}^{i} u_{y y}^{j} \\
& v_{t}^{\alpha}=v^{\alpha} v_{y}^{\alpha}+w_{y}+\frac{\epsilon}{2}\left(u^{1}+\cdots+u^{n}\right)_{y y}+\frac{\epsilon}{2} D_{\beta}^{\alpha} v_{y y}^{\beta} \\
& \triangle w=\frac{T+1}{2}\left(\sum_{k=1}^{n} u_{y}^{k}-\sum_{\alpha=1}^{m} v_{y}^{\alpha}\right)
\end{aligned}
$$

where $D_{j}^{i}$ and $D_{\beta}^{\alpha}$ are the $n \times n$ and $m \times m$ skew-symmetric matrices with 1 's and -1 's above/below the main diagonal. The corresponding Lax pair is

$$
\prod_{\alpha=1}^{m}\left(\epsilon \partial_{y}-v^{\alpha}\right) T \psi=\prod_{k=1}^{n}\left(\epsilon \partial_{y}-u^{k}\right) \psi, \quad \epsilon \psi_{t}=\frac{\epsilon^{2}}{2} \psi_{y y}+\left(w+\frac{\epsilon}{2}\left(\sum_{\alpha=1}^{m} v_{y}^{\alpha}-\sum_{k=1}^{n} u_{y}^{k}\right)\right) \psi .
$$

We believe that system (15) plays the role of a 'master integrable system' in the classification of multi-component

\begin{tabular}{|c|c|c|}
\hline$E q n$ & Lax pair & Dispersionless Lax pair \\
\hline (9) & $\begin{array}{l}2 \epsilon T \psi_{y}=-2 \epsilon \psi_{y}+(v-u) T \psi+(u+v) \psi \\
\epsilon \psi_{t}=\epsilon^{2} \psi_{y y}+\left(2 w-\epsilon u_{y}\right) \psi\end{array}$ & $\begin{array}{l}e^{S_{x}}=\frac{u+v-2 S_{y}}{u-v+2 S_{y}} \\
S_{t}=S_{y}^{2}+2 w\end{array}$ \\
\hline (10) & $\begin{array}{l}\epsilon T \psi_{y}=e^{-u} v \psi-\epsilon e^{-u} \psi_{y} \\
\psi_{t}=\frac{\epsilon}{2} \psi_{y y}+\left(w-\frac{\epsilon}{2} u_{y}\right) \psi_{y}\end{array}$ & $\begin{array}{l}e^{S_{x}}=\frac{e^{-u}\left(v-S_{y}\right)}{S_{y}} \\
S_{t}=\frac{1}{2} S_{y}^{2}+w S_{y}\end{array}$ \\
\hline (11) & $\begin{array}{l}\epsilon T \psi_{y}=2 \epsilon e^{2 u} \psi_{y}-2 v e^{u} \operatorname{sech} u(T \psi+2 \psi) \\
\psi_{t}=-\frac{\epsilon}{4} \psi_{y y}+\left(w-\frac{\epsilon}{2} u_{y}\right) \psi_{y}\end{array}$ & $\begin{array}{l}e^{S_{x}}=2 e^{2 u} \frac{S_{y}-2 e^{-u} v \operatorname{sech} u}{S_{y}+2 e^{u} v \operatorname{sech} u} \\
S_{t}=-\frac{1}{4} S_{y}^{2}+w S_{y}\end{array}$ \\
\hline (12) & $\begin{array}{l}T \psi=\frac{\epsilon^{2}}{4} \psi_{y y}-\epsilon u \psi_{y}+\left(u^{2}-\frac{\epsilon}{2} u_{y}-\frac{\epsilon}{2} v_{y}-v^{2}\right) \psi \\
\epsilon \psi_{t}=\frac{\epsilon^{2}}{4} \psi_{y y}+\left(2 w-\epsilon u_{y}\right) \psi\end{array}$ & $\begin{array}{l}e^{S_{x}}=\frac{1}{4} S_{y}^{2}-u S_{y}+u^{2}-v^{2} \\
S_{t}=\frac{1}{4} S_{y}^{2}+2 w\end{array}$ \\
\hline (13) & $\begin{array}{l}T \psi=\frac{\epsilon^{2}}{4} \psi_{y y}-\epsilon u \psi_{y}+\left(u^{2}-\frac{\epsilon}{2}(1+i) u_{y}-2 v\right) \psi \\
\epsilon \psi_{t}=\frac{\epsilon^{2}}{4} \psi_{y y}+\left(2 w-\epsilon u_{y}\right) \psi\end{array}$ & $\begin{array}{l}e^{S_{x}}=\frac{1}{4} S_{y}^{2}-u S_{y}+u^{2}-2 v \\
S_{t}=\frac{1}{4} S_{y}^{2}+2 w\end{array}$ \\
\hline (14) & $\begin{array}{l}T \psi=\frac{1}{2} \epsilon^{2} e^{-2 u} \psi_{y y}-\frac{1}{2} \epsilon^{2} e^{-2 u}\left(u_{y}+\frac{v_{y}}{\sqrt{v^{2}+\alpha}}\right) \psi_{y}-2 \epsilon v e^{-u} \psi_{y}-2 \alpha \psi \\
\psi_{t}=\frac{\epsilon}{4} \psi_{y y}+\left(w-\frac{\epsilon}{2} u_{y}\right) \psi_{y}\end{array}$ & $\begin{array}{l}e^{S_{x}}=\frac{1}{2} e^{-2 u} S_{y}^{2}-2 v e^{-u} S_{y}-2 \alpha \\
S_{t}=\frac{1}{4} S_{y}^{2}+w S_{y}\end{array}$ \\
\hline
\end{tabular}
integrable equations with nonlocality of ILW type.

Lax pairs for systems from Theorem 2 are provided in the table below. They are obtained as quantisations of dispersionless Lax pairs [20] (which are presented in the form that makes the quantisation procedure intuitively clear).

\section{Proof of Theorem 2}

Our approach is based on a two-step procedure which can be summarised as follows: 
- First, we consider dispersionless limit of system (8) and require that the corresponding Haantjes tensor vanishes [10]. This gives necessary conditions for integrability in the form of differential constraints for the coefficients $\alpha, \beta, \gamma, \phi, \psi, \eta$, leading to the two essentially different cases, see details below.

- Secondly, we require that all one-phase hydrodynamic reductions (simple waves) of the dispersionless limits obtained at the previous step can be deformed into reductions of the full dispersive system (8). This approach, which can be seen as a $2+1$ dimensional extension of the deformation procedure developed by the Dubrovin school in $1+1$ dimensions [5, 6], has been proposed in [8, 9] and successfully adapted to the classification of integrable systems in $2+1$ dimensions, see [17, 16, 11, 12. Applied to system (8), it results in further constraints for the dispersionless limit and $\epsilon$-corrections, eventually leading to the six cases of Theorem 2 ,

Let us go through the above scheme in some more detail. The dispersionless limit of system (8) is

$$
\begin{aligned}
& u_{t}=\alpha(u, v, w) u_{y}+\beta(u, v, w) v_{y}+\gamma(u, v, w) w_{y} \\
& v_{t}=\phi(u, v, w) u_{y}+\psi(u, v, w) v_{y}+\eta(u, v, w) w_{y} \\
& w_{x}=u_{y}
\end{aligned}
$$

Introducing the column vector $U=\left(u^{1}, u^{2}, u^{3}\right)^{T}=(u, v, w)^{T}$, we can represent system (16) in matrix form,

$$
A U_{t}+B U_{x}+C U_{y}=0
$$

where the $3 \times 3$ matrices $A, B$ and $C$ are as follows:

$$
A=\left(\begin{array}{ccc}
-1 & 0 & 0 \\
0 & -1 & 0 \\
0 & 0 & 0
\end{array}\right), \quad B=\left(\begin{array}{ccc}
0 & 0 & 0 \\
0 & 0 & 0 \\
0 & 0 & -1
\end{array}\right), \quad C=\left(\begin{array}{ccc}
\alpha & \beta & \gamma \\
\phi & \psi & \eta \\
1 & 0 & 0
\end{array}\right)
$$

The dispersion relation of system (17) is defined by the formula $\operatorname{det}(\lambda A+\mu B+C)=0$, which gives a rational cubic curve:

$$
\mu((\alpha-\lambda)(\psi-\lambda)-\beta \phi)+\gamma(\phi-\lambda)-\beta \eta=0 .
$$

In what follows we will assume that the dispersion relation defines an irreducible cubic.

Let us introduce the matrix $V=(B+p A)^{-1}(C+q A)$ where $p$ and $q$ are arbitrary parameters. It was demonstrated in [10] that the necessary condition for integrability of the dispersionless limit (16) is the vanishing of the Haantjes tensor [15] of the matrix $V$, identically in the parameters $p, q$. Recall that, given a matrix $V$, its Haantjes tensor is defined by the formula

$$
H_{j k}^{i}=N_{p r}^{i} V_{j}^{p} V_{k}^{r}-N_{j r}^{p} V_{p}^{i} V_{k}^{r}-N_{r k}^{p} V_{p}^{i} V_{j}^{r}+N_{j k}^{p} V_{r}^{i} V_{p}^{r}
$$

where

$$
N_{j k}^{i}=V_{j}^{p} \partial_{u^{p}} V_{k}^{i}-V_{k}^{p} \partial_{u^{p}} V_{j}^{i}-V_{p}^{i}\left(\partial_{u^{j}} V_{k}^{p}-\partial_{u^{k}} V_{j}^{p}\right)
$$

is the Nijenhuis tensor. The requirement of vanishing of the Haantjes tensor imposes strong constraints on the coefficients of system (16). One of them is the relation

$$
\gamma \eta_{w}=\eta \gamma_{w}
$$

which splits the further analysis into two cases:

Case 1: $\gamma=0$.

Case 2: $\gamma \neq 0$. In this case we have $\eta=s(u, v) \gamma$. Utilising the transformation freedom $v \rightarrow f(u, v)$, we can set $\eta=0$.

Let us discuss Case 1 in some more detail (Case 2 can be treated analogously). If $\gamma=0$, the vanishing of the Haantjes tensor implies further constraints such as

$$
\beta_{w}=\phi_{w}=\eta_{w}=(\alpha-\psi)_{w}=0
$$

etc. In particular, $\beta=b(u, v)$, and utilising the transformation freedom $v \rightarrow f(u, v)$, we can set $\beta=1$; note that $\beta$ cannot vanish, otherwise, the dispersion relation (18) would become reducible. Further analysis of the Haantjes tensor leads to the two subcases. 
Subcase 1.1:

$$
\begin{aligned}
& u_{t}=-\frac{1}{2}\left(\alpha+u \alpha^{\prime}\right) u_{y}+v_{y} \\
& v_{t}=\left(-\frac{v^{2}}{u^{2}}+a u^{2}-v \alpha^{\prime}-\frac{1}{4} u^{2} \alpha^{\prime 2}\right) u_{y}+\left(2 \frac{v}{u}-\frac{1}{2} \alpha+\frac{1}{2} u \alpha^{\prime}\right) v_{y}+b u w_{y}, \\
& w_{x}=u_{y}
\end{aligned}
$$

where $\alpha(u)$ is an arbitrary function and $a, b$ are arbitrary constants. The corresponding Haantjes tensor vanishes identically. Note that the arbitrary function $\alpha(u)$ can be eliminated by the substitution $v \rightarrow v+\frac{1}{2} u \alpha(u)$, leading to the simplified form

$$
\begin{aligned}
& u_{t}=v_{y} \\
& v_{t}=\left(-\frac{v^{2}}{u^{2}}+a u^{2}\right) u_{y}+\frac{v}{u} v_{y}+b u w_{y}, \\
& w_{x}=u_{y}
\end{aligned}
$$

(particular values of constants $a, b$ are not important, we will normalise them at a later stage).

Subcase 1.2:

$$
\begin{aligned}
& u_{t}=\left(c w+\alpha^{\prime}\right) u_{y}+v_{y}, \\
& v_{t}=\left(-\frac{1}{2} c v^{2} \eta^{\prime}-c v \alpha \eta^{\prime}-\frac{1}{2} c \alpha^{2} \eta^{\prime}-c v \eta \alpha^{\prime}-c \alpha \eta \alpha^{\prime}-\alpha^{\prime 2}\right) u_{y}+\left(c w-c v \eta-c \alpha \eta-\alpha^{\prime}\right) v_{y}+c(v+\alpha) w_{y}, \\
& w_{x}=u_{y}
\end{aligned}
$$

where $\alpha(u)$ is an arbitrary function, $c$ is an arbitrary constant and the function $\eta(u)$ satisfies the second-order ODE, $\eta^{\prime \prime}=c \eta \eta^{\prime}$. The corresponding Haantjes tensor vanishes identically. Again, the arbitrary function $\alpha(u)$ can be eliminated by the substitution $v \rightarrow v-\alpha(u)$, leading to the simplified form

$$
\begin{aligned}
& u_{t}=c w u_{y}+v_{y}, \\
& v_{t}=-\frac{1}{2} c v^{2} \eta^{\prime} u_{y}+c(w-v \eta) v_{y}+c v w_{y}, \\
& w_{x}=u_{y} .
\end{aligned}
$$

Note that one can scale the constant to $c=1$, then ODE $\eta^{\prime \prime}=\eta \eta^{\prime}$ leads to the two further subcases: $\eta=1$ and $\eta=-2 \tanh u$.

To summarise, based on the requirement of vanishing of the Haantjes tensor only, in Case 1 we have found three candidates for dispersionless limits of integrable systems (8), namely, systems (19) and (20) (for two different forms of $\eta$ ). The next step of the classification is to consider one-phase reductions of these dispersionless systems and require that they can be deformed into reductions of the corresponding dispersive systems (8).

This step is computationally intense, the details are as follows. Recall that one-phase reductions (simple waves) of system (17) are defined by the formula $U=U(R)$ where the 'phase' $R$ satisfies a pair of compatible equations

$$
R_{t}=\lambda(R) R_{y}, \quad R_{x}=\mu(R) R_{y}
$$

Substituting this ansatz into (17) we obtain the relation

$$
(\lambda A+\mu B+C) U^{\prime}=0
$$

where $U^{\prime}=d U / d R$. Thus, $U^{\prime}$ is the eigenvector of the matrix $\lambda A+\mu B+C$, while the characteristic speeds $\lambda, \mu$ satisfy the dispersion relation, $\operatorname{det}(\lambda A+\mu B+C)=0$. Let us look for solutions of system (8) in the form

$$
U=U(R)+\epsilon(\ldots)+\epsilon^{2}(\ldots)+\ldots
$$

where dots at $\epsilon^{n}$ denote differential polynomials in $y$-derivatives of $R$ of the total degree $n$, whose coefficients are some functions of $R$ (expansions of this kind do not terminate in general). Similarly, we assume that equations (21) are also deformed,

$$
R_{t}=\lambda(R) R_{y}+\epsilon(\ldots)+\epsilon^{2}(\ldots)+\ldots, \quad R_{x}=\mu(R) R_{y}+\epsilon(\ldots)+\epsilon^{2}(\ldots)+\ldots
$$

here dots at $\epsilon^{n}$ denote differential polynomials in $y$-derivatives of $R$ of the total degree $n+1$. Let us substitute (22) into (8). Using (23) and requiring that the terms at the same powers of $\epsilon$ vanish identically, we can explicitly reconstruct the expansions (22) and (23), where all terms denoted by dots can be uniquely expressed in terms of $U(R), \lambda(R), \mu(R)$, and the $\epsilon$-terms in equation (8). Furthermore, by looking at terms of the order not higher 
than $\epsilon^{2}$, we get strong necessary conditions for the $\epsilon$-terms in (8) which, in this classification problem, turn out to be also sufficient (this can be demonstrated by directly constructing the Lax pairs).

Applied to dispersionless limit (19), the above procedure leads to case (9) of Theorem 2 (up to the change of variables $v \rightarrow u v$ and suitable rescalings).

Similarly, applied to dispersionless limit (20), the above procedure leads to cases (10) and (11) of Theorem 2 (which correspond to $\eta=1$ and $\eta=-2 \tanh u$, respectively).

Similarly, Case 2 leads to systems (12), (13) and (14).

\section{$3 \quad$ Waterbag system in $2+1$ dimensions}

Several equations appearing in Theorems 1+2 can be obtained, as special cases, from the $2+1$ dimensional dispersionless integrable system

$$
u_{t}^{i}=u^{i} u_{y}^{i}+w_{y}, \quad w_{x}=\sum_{k=1}^{N} \frac{1}{\omega_{k}} u_{y}^{k},
$$

$i=1, \ldots, N$, which possesses the Lax pair

$$
e^{S_{x}}=\prod_{k=1}^{N}\left(S_{y}-u^{k}\right)^{\frac{1}{\omega_{k}}}, \quad S_{t}=\frac{1}{2} S_{y}^{2}+w
$$

here $\omega_{k}$ are arbitrary constants. In the $1+1$ dimensional limit, $y=-x$, system (24) reduces to

$$
u_{t}^{i}+u^{i} u_{x}^{i}-\sum_{k=1}^{N} \frac{1}{\omega_{k}} u_{x}^{k}=0,
$$

the so-called 'waterbag' reduction of the Benney chain [13. It was shown in [3] that a special case of system (25) describes propagation of long nonlinear waves in a shear flow with piecewise linear velocity profile, see Section 4

Dispersive integrable extension of system (24) is not known (for generic $\omega_{k}$ ). However, for special values $\omega_{k}= \pm 1$, the corresponding dispersive extensions are associated with Lax pairs of the form

$$
P\left(\epsilon \partial_{y}\right) T \psi=Q\left(\epsilon \partial_{y}\right) \psi, \quad \epsilon \psi_{t}=\frac{\epsilon^{2}}{2} \psi_{y y}+w \psi
$$

where $P$ and $Q$ are differential operators whose coefficients depend on $u$ 's. Below we discuss some special cases of this construction.

\subsection{Case $N=1, \omega_{1}=1$}

The corresponding system (24) takes the form

$$
u_{t}=u u_{y}+w_{y}, \quad w_{x}=u_{y}
$$

with the dispersionless Lax pair

$$
e^{S_{x}}=S_{y}-u, \quad S_{t}=\frac{1}{2} S_{y}^{2}+w
$$

This system possesses dispersive deformation (the first case of Theorem 1):

$$
u_{t}=u u_{y}+w_{y}, \quad \triangle w=\frac{T+1}{2} u_{y}
$$

with the Lax pair

$$
T \psi=\epsilon \psi_{y}-u \psi, \quad \epsilon \psi_{t}=\frac{\epsilon^{2}}{2} \psi_{y y}+\left(w-\frac{\epsilon}{2} u_{y}\right) \psi
$$


3.2 Case $N=2, \omega_{1}=1, \omega_{2}=-1$

The corresponding system (24) takes the form

$$
u_{t}=u u_{y}+w_{y}, \quad v_{t}=v v_{y}+w_{y}, \quad w_{x}=(u-v)_{y},
$$

with the dispersionless Lax pair

$$
e^{S_{x}}=\frac{S_{y}-u}{S_{y}-v}, \quad S_{t}=\frac{1}{2} S_{y}^{2}+w .
$$

System (26) possesses the following dispersive deformation:

$$
u_{t}=u u_{y}+w_{y}+\frac{\epsilon}{2} v_{y y}, \quad v_{t}=v v_{y}+w_{y}+\frac{\epsilon}{2} u_{y y}, \quad \triangle w=\frac{T+1}{2}(u-v)_{y},
$$

with the Lax pair

$$
\left(\epsilon \partial_{y}-v\right) T \psi=\left(\epsilon \partial_{y}-u\right) \psi, \quad \epsilon \psi_{t}=\frac{\epsilon^{2}}{2} \psi_{y y}+\left(w+\frac{\epsilon}{2}(v-u)_{y}\right) \psi
$$

Note that system (27) coincides with (9) in the variables $\hat{u}=u-v, \hat{v}=u+v$ (up to unessential scaling factors). System (27) is equivalent to the differential-difference Davey-Stewartson (DS) equation. To see this, we rewrite Lax pair (28) in equivalent form,

$$
T \psi=\left(1+\frac{1}{\epsilon} p \partial_{y}^{-1} q\right) \psi, \quad \epsilon \psi_{t}=\frac{\epsilon^{2}}{2} \psi_{y y}+\tilde{w} \psi,
$$

where the new variables $p, q, \tilde{w}$ are connected to $u, v, w$ by the formulae

$$
u=\epsilon \frac{p_{y}}{p}-p q, \quad v=\epsilon \frac{p_{y}}{p}, \quad w=\tilde{w}-\frac{\epsilon}{2}(v-u)_{y} .
$$

The compatibility conditions of Lax pair (29) result in the differential-difference DS equation,

$$
\epsilon p_{t}=\frac{\epsilon^{2}}{2} p_{y y}+p T \tilde{w}, \quad-\epsilon q_{t}=\frac{\epsilon^{2}}{2} q_{y y}+q \tilde{w}, \quad \triangle \tilde{w}=-(p q)_{y} .
$$

Remark. Lax pair (29) and system (30) possess natural multi-component extensions:

$$
T \psi=\left(1+\frac{1}{\epsilon} \sum_{k} p^{k} \partial_{y}^{-1} q^{k}\right) \psi, \quad \epsilon \psi_{t}=\frac{\epsilon^{2}}{2} \psi_{y y}+\tilde{w} \psi,
$$

and

$$
\epsilon p_{t}^{i}=\frac{\epsilon^{2}}{2} p_{y y}^{i}+p^{i} T \tilde{w}, \quad-\epsilon q_{t}^{i}=\frac{\epsilon^{2}}{2} q_{y y}^{i}+q^{i} \tilde{w}, \quad \triangle \tilde{w}=-\left(\sum_{k} p^{k} q^{k}\right)_{y},
$$

respectively. This multi-component differential-difference DS system is apparently new. Applying the Madelung transformation,

$$
p^{i}=\sqrt{\eta^{i}} e^{\frac{1}{\epsilon} \int u^{i} d y}, \quad q^{i}=\sqrt{\eta^{i}} e^{-\frac{1}{\epsilon} \int u^{i} d y},
$$

one can rewrite system (32) in the Hasimoto form,

$$
\eta_{t}^{i}=\left(u^{i} \eta^{i}\right)_{y}+\eta^{i} \triangle \tilde{w}, \quad u_{t}^{i}=u^{i} u_{y}^{i}+\frac{T+1}{2} \tilde{w}_{y}+\frac{\epsilon^{2}}{4}\left(\frac{\eta_{y y}^{i}}{\eta^{i}}-\frac{1}{2} \frac{\left(\eta_{y}^{i}\right)^{2}}{\left(\eta^{i}\right)^{2}}\right)_{y}, \quad \triangle \tilde{w}=-\left(\sum_{k} \eta^{k}\right)_{y} .
$$

Introducing the new variables $v^{i}$ by the formula $v^{i}=u^{i}-\frac{\epsilon}{2}\left(\ln \eta^{i}\right)_{y}$ we obtain the equivalent Kaup-Broer form:

$$
\eta_{t}^{i}=\left(v^{i} \eta^{i}\right)_{y}+\eta^{i} \triangle \tilde{w}+\frac{\epsilon}{2} \eta_{y y}^{i}, \quad v_{t}^{i}=v^{i} v_{y}^{i}+\frac{T+1}{2} \tilde{w}_{y}-\frac{\epsilon}{2}\left(v_{y}^{i}+\triangle \tilde{w}\right)_{y}, \quad \triangle \tilde{w}=-\left(\sum_{k} \eta^{k}\right)_{y}
$$


3.3 Case $N=2, \omega_{1}=-\omega_{2}$

Setting $\omega_{1}=-\omega_{2}=\omega$, one can rescale this case back to the previous one by setting $x=\omega \tilde{x}$. However, our goal here is to investigate the limit $\omega \rightarrow 0$. Thus, system (27) and its Lax pair (28) assume the form

$$
u_{t}=u u_{y}+w_{y}+\frac{\epsilon}{2} v_{y y}, \quad v_{t}=v v_{y}+w_{y}+\frac{\epsilon}{2} u_{y y}, \quad \frac{\tilde{T}-1}{\epsilon} w=\frac{\tilde{T}+1}{2}(u-v)_{y}
$$

and

$$
\left(\epsilon \partial_{y}-v\right) \tilde{T} \psi=\left(\epsilon \partial_{y}-u\right) \psi, \quad \epsilon \psi_{t}=\frac{\epsilon^{2}}{2} \psi_{y y}+\left(w+\frac{\epsilon}{2}(v-u)_{y}\right) \psi
$$

respectively; here $\tilde{T}=e^{\epsilon \partial_{\tilde{x}}}=e^{\epsilon \omega \partial_{x}}$. Setting $u=v-\omega \eta$, we can rewrite (33) and (34) in the form

$$
\eta_{t}=(v \eta)_{y}-\omega \eta \eta_{y}-\frac{\epsilon}{2} \eta_{y y}, \quad v_{t}=v v_{y}+w_{y}+\frac{\epsilon}{2} v_{y y}-\frac{\epsilon \omega}{2} \eta_{y y}, \quad \frac{\tilde{T}-1}{\epsilon \omega} w=-\frac{\tilde{T}+1}{2} \eta_{y}
$$

and

$$
\epsilon\left(\epsilon \partial_{y}-v\right) \frac{\tilde{T}-1}{\epsilon \omega} \psi=\eta \psi, \quad \epsilon \psi_{t}=\frac{\epsilon^{2}}{2} \psi_{y y}+\left(w+\frac{\epsilon \omega}{2} \eta_{y}\right) \psi
$$

respectively. In the limit $\omega \rightarrow 0$ we have $\frac{\tilde{T}-1}{\epsilon \omega} \rightarrow \partial_{x}$, which results in the system

$$
\eta_{t}=(v \eta)_{y}-\frac{\epsilon}{2} \eta_{y y}, \quad v_{t}=v v_{y}+w_{y}+\frac{\epsilon}{2} v_{y y}, \quad w_{x}=-\eta_{y}
$$

and its Lax pair,

$$
\epsilon\left(\epsilon \partial_{y}-v\right) \psi_{x}=\eta \psi, \quad \epsilon \psi_{t}=\frac{\epsilon^{2}}{2} \psi_{y y}+w \psi
$$

see [20], Section 3 .

\subsection{Case $N=2, \omega_{1}=\omega_{2}=1$}

The corresponding system (24) takes the form

$$
u_{t}=u u_{y}+w_{y}, \quad v_{t}=v v_{y}+w_{y}, \quad w_{x}=(u+v)_{y},
$$

with the dispersionless Lax pair

$$
e^{S_{x}}=\left(S_{y}-u\right)\left(S_{y}-v\right), \quad S_{t}=\frac{1}{2} S_{y}^{2}+w .
$$

System (36) possesses dispersive deformation

$$
u_{t}=u u_{y}+w_{y}+\frac{\epsilon}{2} v_{y y}, \quad v_{t}=v v_{y}+w_{y}-\frac{\epsilon}{2} u_{y y}, \quad \triangle w=\frac{T+1}{2}(u+v)_{y},
$$

with the Lax pair

$$
T \psi=\left(\epsilon \partial_{y}-u\right)\left(\epsilon \partial_{y}-v\right) \psi, \quad \epsilon \psi_{t}=\frac{\epsilon^{2}}{2} \psi_{y y}+\left(w-\frac{\epsilon}{2}(u+v)_{y}\right) \psi
$$

Up to elementary changes of variables, this case is equivalent to systems (12), (13): this can be seen from the structure of the corresponding Lax pairs. Interestingly, system (36) possesses yet another (third-order) dispersive deformation,

$$
u_{t}=u u_{y}+w_{y}-\frac{\epsilon^{2}}{4} \frac{u_{y y y}+v_{y y y}}{u-v}, \quad v_{t}=v v_{y}+w_{y}-\frac{\epsilon^{2}}{4} \frac{u_{y y y}+v_{y y y}}{v-u}, \quad \triangle w=\frac{T+1}{2}(u+v)_{y},
$$

associated with the 'symmetrised' Lax pair,

$$
T \psi=\left(\epsilon^{2} \partial_{y}^{2}-\epsilon(u+v) \partial_{y}+u v-\frac{\epsilon}{2}(u+v)_{y}\right) \psi, \quad \epsilon \psi_{t}=\frac{\epsilon^{2}}{2} \psi_{y y}+\left(w-\frac{\epsilon}{2}(u+v)_{y}\right) \psi
$$

Both dispersive deformations are clearly Miura-equivalent. 
3.5 Case $N=n, \omega_{1}=\cdots=\omega_{n}=1$

Let us begin with the 3-component case,

$$
u_{t}^{i}=u^{i} u_{y}^{i}+w_{y}, \quad w_{x}=\left(u^{1}+u^{2}+u^{3}\right)_{y},
$$

$i=1,2,3$, with the dispersionless Lax pair

$$
e^{S_{x}}=\left(S_{y}-u^{1}\right)\left(S_{y}-u^{2}\right)\left(S_{y}-u^{3}\right), \quad S_{t}=\frac{1}{2} S_{y}^{2}+w .
$$

System (36) possesses dispersive deformation

$$
\begin{gathered}
u_{t}^{1}=u^{1} u_{y}^{1}+w_{y}+\frac{\epsilon}{2}\left(u^{2}+u^{3}\right)_{y y}, \\
u_{t}^{2}=u^{2} u_{y}^{2}+w_{y}+\frac{\epsilon}{2}\left(-u^{1}+u^{3}\right)_{y y}, \\
u_{t}^{3}=u^{3} u_{y}^{3}+w_{y}+\frac{\epsilon}{2}\left(-u^{1}-u^{2}\right)_{y y}, \\
\triangle w=\frac{T+1}{2}\left(u^{1}+u^{2}+u^{3}\right)_{y},
\end{gathered}
$$

with the Lax pair

$$
T \psi=\left(\epsilon \partial_{y}-u^{1}\right)\left(\epsilon \partial_{y}-u^{2}\right)\left(\epsilon \partial_{y}-u^{3}\right) \psi, \quad \epsilon \psi_{t}=\frac{\epsilon^{2}}{2} \psi_{y y}+\left(w-\frac{\epsilon}{2}\left(u^{1}+u^{2}+u^{3}\right)_{y}\right) \psi .
$$

The general $n$-component version is as follows:

$$
\begin{gathered}
u_{t}^{i}=u^{i} u_{y}^{i}+w_{y}+\frac{\epsilon}{2} D_{j}^{i} u_{y y}^{j}, \\
\triangle w=\frac{T+1}{2} \sum u_{y}^{k},
\end{gathered}
$$

where $D_{j}^{i}$ is the $n \times n$ skew-symmetric matrix with 1's and -1 's above/below the main diagonal. The corresponding Lax pair is

$$
T \psi=\prod\left(\epsilon \partial_{y}-u^{k}\right) \psi, \quad \epsilon \psi_{t}=\frac{\epsilon^{2}}{2} \psi_{y y}+\left(w-\frac{\epsilon}{2} \sum u_{y}^{k}\right) \psi
$$

3.6 Case $N=2 n, \omega_{1}=\cdots=\omega_{n}=1, \quad \omega_{n+1}=\cdots=\omega_{2 n}=-1$

The corresponding system (24) takes the form (we set $v^{i}=u^{n+i}$ ):

$$
u_{t}^{i}=u^{i} u_{y}^{i}+w_{y}, \quad v_{t}^{i}=v^{i} v_{y}^{i}+w_{y}, \quad w_{x}=\sum_{k=1}^{n}\left(u^{k}-v^{k}\right)_{y} .
$$

It possesses dispersionless Lax pair

$$
e^{S_{x}}=\prod_{k=1}^{n} \frac{S_{y}-u^{k}}{S_{y}-v^{k}}, \quad S_{t}=\frac{1}{2} S_{y}^{2}+w,
$$

which has dispersive extension of the form

$$
P\left(\epsilon \partial_{y}\right) T \psi=Q\left(\epsilon \partial_{y}\right) \psi, \quad \epsilon \psi_{t}=\frac{\epsilon^{2}}{2} \psi_{y y}+w \psi ;
$$

here $P$ and $Q$ are differential operators of degree $n$ whose coefficients depend on $u$ 's and $v$ 's. This Lax pair and the associated dispersive system are Miura-equivalent to (31), (32). As an example let us consider the case $N=4$. The Lax pair

$$
\begin{gathered}
\left(\epsilon \partial_{y}-v^{1}\right)\left(\epsilon \partial_{y}-v^{2}\right) T \psi=\left(\epsilon \partial_{y}-u^{1}\right)\left(\epsilon \partial_{y}-u^{2}\right) \psi, \\
\epsilon \psi_{t}=\frac{\epsilon^{2}}{2} \psi_{y y}+\left(w+\frac{\epsilon}{2}\left(v^{1}+v^{2}-u^{1}-u^{2}\right)_{y}\right) \psi
\end{gathered}
$$


results in the second-order deformation,

$$
\begin{gathered}
u_{t}^{1}=u^{1} u_{y}^{1}+w_{y}+\frac{\epsilon}{2}\left(v^{1}+v^{2}+u^{2}\right)_{y y}, \\
u_{t}^{2}=u^{2} u_{y}^{2}+w_{y}+\frac{\epsilon}{2}\left(v^{1}+v^{2}-u^{1}\right)_{y y}, \\
v_{t}^{1}=v^{1} v_{y}^{1}+w_{y}+\frac{\epsilon}{2}\left(u^{1}+u^{2}+v^{2}\right)_{y y}, \\
v_{t}^{2}=v^{2} v_{y}^{2}+w_{y}+\frac{\epsilon}{2}\left(u^{1}+u^{2}-v^{1}\right)_{y y}, \\
\triangle w=\frac{T+1}{2}\left(u^{1}+u^{2}-v^{1}-v^{2}\right)_{y} .
\end{gathered}
$$

The 'symmetrised' Lax pair,

$$
\begin{gathered}
\left(\left(\epsilon \partial_{y}-v^{1}\right)\left(\epsilon \partial_{y}-v^{2}\right)+\left(\epsilon \partial_{y}-v^{2}\right)\left(\epsilon \partial_{y}-v^{1}\right)\right) T \psi=\left(\left(\epsilon \partial_{y}-u^{1}\right)\left(\epsilon \partial_{y}-u^{2}\right)+\left(\epsilon \partial_{y}-u^{2}\right)\left(\epsilon \partial_{y}-u^{1}\right)\right) \psi, \\
\epsilon \psi_{t}=\frac{\epsilon^{2}}{2} \psi_{y y}+\left(w+\frac{\epsilon}{2}\left(v^{1}+v^{2}-u^{1}-u^{2}\right)_{y}\right) \psi,
\end{gathered}
$$

results in the third-order deformation,

$$
\begin{gathered}
u_{t}^{1}=u^{1} u_{y}^{1}+w_{y}+\frac{\epsilon}{2}\left(v_{y y}^{1}+v_{y y}^{2}\right)-\frac{\epsilon^{2}}{4} \frac{u_{y y y}^{1}+u_{y y y}^{2}}{u^{1}-u^{2}}, \\
u_{t}^{2}=u^{2} u_{y}^{2}+w_{y}+\frac{\epsilon}{2}\left(v_{y y}^{1}+v_{y y}^{2}\right)-\frac{\epsilon^{2}}{4} \frac{u_{y y y}^{1}+u_{y y y}^{2}}{u^{2}-u^{1}}, \\
v_{t}^{1}=v^{1} v_{y}^{1}+w_{y}+\frac{\epsilon}{2}\left(u_{y y}^{1}+u_{y y}^{2}\right)-\frac{\epsilon^{2}}{4} \frac{v_{y y y}^{1}+v_{y y y}^{2}}{v^{1}-v^{2}}, \\
v_{t}^{2}=v^{2} v_{y}^{2}+w_{y}+\frac{\epsilon}{2}\left(u_{y y}^{1}+u_{y y}^{2}\right)-\frac{\epsilon^{2}}{4} \frac{v_{y y y}^{1}+v_{y y y}^{2}}{v^{2}-v^{1}}, \\
\triangle w=\frac{T+1}{2}\left(u^{1}+u^{2}-v^{1}-v^{2}\right)_{y} .
\end{gathered}
$$

Both second-order and third-order deformations are Miura-equivalent.

The general $2 n$-component version is as follows:

$$
\begin{gathered}
u_{t}^{i}=u^{i} u_{y}^{i}+w_{y}+\frac{\epsilon}{2}\left(v^{1}+\cdots+v^{n}\right)_{y y}+\frac{\epsilon}{2} D_{j}^{i} u_{y y}^{j}, \\
v_{t}^{i}=v^{i} v_{y}^{i}+w_{y}+\frac{\epsilon}{2}\left(u^{1}+\cdots+u^{n}\right)_{y y}+\frac{\epsilon}{2} D_{j}^{i} v_{y y}^{j} \\
\triangle w=\frac{T+1}{2} \sum\left(u^{k}-v^{k}\right)_{y},
\end{gathered}
$$

where $D_{j}^{i}$ is the $n \times n$ skew-symmetric matrix with 1's and -1 's above/below the main diagonal. The corresponding Lax pair is

$$
\prod\left(\epsilon \partial_{y}-v^{k}\right) T \psi=\prod\left(\epsilon \partial_{y}-u^{k}\right) \psi, \quad \epsilon \psi_{t}=\frac{\epsilon^{2}}{2} \psi_{y y}+\left(w+\frac{\epsilon}{2} \sum\left(v^{k}-u^{k}\right)_{y}\right) \psi
$$

3.7 Case $N=2 n, \omega_{n+i}=-\omega_{i}$

Setting $u^{n+i}=v^{i}$ one can write the corresponding equations in the form

$$
u_{t}^{i}=u^{i} u_{y}^{i}+w_{y}, \quad v_{t}^{i}=v^{i} v_{y}^{i}+w_{y}, \quad w_{x}=\sum_{k=1}^{n} \frac{u_{y}^{k}-v_{y}^{k}}{\omega_{k}} .
$$

This system possesses dispersionless Lax pair

$$
e^{S_{x}}=\prod_{k=1}^{n}\left(\frac{S_{y}-u^{k}}{S_{y}-v^{k}}\right)^{\frac{1}{\omega_{k}}}, \quad S_{t}=\frac{1}{2} S_{y}^{2}+w
$$


equivalently,

$$
S_{x}=\sum_{k=1}^{n} \frac{1}{\omega_{k}} \ln \frac{S_{y}-u^{k}}{S_{y}-v^{k}}, \quad S_{t}=\frac{1}{2} S_{y}^{2}+w .
$$

System (39) can be viewed as a $2+1$ dimensional generalisation of the $1+1$ dimensional system governing shear flow in $n$-layered fluid, see [3] and Section 4 of this paper. Setting $u^{k}=v^{k}-\omega_{k} \eta^{k}$, we can rewrite (39) in the form

$$
v_{t}^{i}=v^{i} v_{y}^{i}+w_{y}, \quad \eta_{t}^{i}=\left(v^{i} \eta^{i}\right)_{y}-\omega_{i} \eta^{i} \eta_{y}^{i}, \quad w_{x}+\sum_{k=1}^{n} \eta_{y}^{k}=0 .
$$

Taking the limit $\omega_{k} \rightarrow 0$ we obtain

$$
S_{x}=\sum_{k=1}^{n} \frac{1}{\omega_{k}} \ln \frac{S_{y}-v^{k}+\omega_{k} \eta^{k}}{S_{y}-v^{k}}=\sum_{k=1}^{n} \frac{1}{\omega_{k}} \ln \left(1+\omega_{k} \frac{\eta^{k}}{S_{y}-v^{k}}\right) \approx \sum_{k=1}^{n} \frac{\eta^{k}}{S_{y}-v^{k}},
$$

which gives the Lax pair

$$
S_{x}=\sum_{k=1}^{n} \frac{\eta^{k}}{S_{y}-v^{k}}, \quad S_{t}=\frac{1}{2} S_{y}^{2}+w
$$

This Lax pair governs the $2+1$ dimensional generalisation of $n$-layer Benney system discussed in 20],

$$
v_{t}^{i}=v^{i} v_{y}^{i}+w_{y}, \quad \eta_{t}^{i}=\left(v^{i} \eta^{i}\right)_{y}, \quad w_{x}+\sum_{k=1}^{n} \eta_{y}^{k}=0,
$$

which is the limit of (38) as $\omega_{k} \rightarrow 0$.

3.8 Case $N=n+m, \omega_{1}=\cdots=\omega_{n}=1, \omega_{n+1}=\cdots=\omega_{n+m}=-1$

This is the most general case where we know the structure of dispersive deformations. Setting $u^{n+\alpha}=v^{\alpha}$ one can write the corresponding equations in the form

$$
u_{t}^{i}=u^{i} u_{y}^{i}+w_{y}, \quad v_{t}^{\alpha}=v^{\alpha} v_{y}^{\alpha}+w_{y}, \quad w_{x}=\sum_{k=1}^{n} u_{y}^{k}-\sum_{\alpha=1}^{m} v_{y}^{\alpha} .
$$

( $n$ variable $u^{i}, m$ variables $\left.v^{\alpha}\right)$. This system possesses integrable dispersive deformation

$$
\begin{gathered}
u_{t}^{i}=u^{i} u_{y}^{i}+w_{y}+\frac{\epsilon}{2}\left(v^{1}+\cdots+v^{m}\right)_{y y}+\frac{\epsilon}{2} D_{j}^{i} u_{y y}^{j}, \\
v_{t}^{\alpha}=v^{\alpha} v_{y}^{\alpha}+w_{y}+\frac{\epsilon}{2}\left(u^{1}+\cdots+u^{n}\right)_{y y}+\frac{\epsilon}{2} D_{\beta}^{\alpha} v_{y y}^{\beta}, \\
\triangle w=\frac{T+1}{2}\left(\sum_{k=1}^{n} u_{y}^{k}-\sum_{\alpha=1}^{m} v_{y}^{\alpha}\right),
\end{gathered}
$$

where $D_{j}^{i}$ and $D_{\beta}^{\alpha}$ are $n \times n$ and $m \times m$ skew-symmetric matrices as in Section 3.6. The corresponding Lax pair is

$$
\prod_{\alpha=1}^{m}\left(\epsilon \partial_{y}-v^{\alpha}\right) T \psi=\prod_{k=1}^{n}\left(\epsilon \partial_{y}-u^{k}\right) \psi, \quad \epsilon \psi_{t}=\frac{\epsilon^{2}}{2} \psi_{y y}+\left(w+\frac{\epsilon}{2}\left(\sum_{\alpha=1}^{m} v_{y}^{\alpha}-\sum_{k=1}^{n} u_{y}^{k}\right)\right) \psi .
$$

\section{Waterbag system and a shear flow with piecewise linear velocity profile}

In this section we discuss a quasilinear system governing multilayer shear flow with piecewise linear velocity profile and constant vorticity within each layer [3]:

$$
u_{t}^{i}+u^{i} u_{x}^{i}+g h_{x}=0, \quad v_{t}^{i}+v^{i} v_{x}^{i}+g h_{x}=0, \quad h=\sum_{k=1}^{n} \frac{v^{k}-u^{k}}{\omega_{k}}
$$


where $v^{i}$ and $u^{i}$ are the velocities at the upper/lower boundary of the $i$-th layer and $\omega_{i}$ is the constant vorticity. It is known that system (40) is strictly hyperbolic, Hamiltonian and possesses Riemann invariants 3 . System (40) can be viewed as an extension of Zakharov's multilayer reduction [22] of the Benney system. It has also appeared in the context of symmetry constraints of the dKP hierarchy [1. We do not impose the continuity condition $v^{i}=u^{i+1}$, generally allowing jumps in horisontal velocities between layers. Such flows could model oceanic and industrial multilayer flows with strongly sheared currents.

One can see that system (40) can be obtained from the $2+1$ dimensional waterbag system (39) by dimensional reduction $y=-x$. The results of Section 3 allow one to construct dispersive regularisations for some special cases of system (40). As the simplest example, let us consider the one-layer case, which is already nontrivial. Setting $u^{1}=u, v^{1}=v$ and normalising $g=\omega_{1}=1$ we obtain the system

$$
u_{t}+u u_{x}+h_{x}=0, \quad v_{t}+v v_{x}+h_{x}=0, \quad h=v-u .
$$

System (41) possesses integrable dispersive regularisation which can be obtained by setting $y=-x$ in equations (27):

$$
u_{t}+u u_{x}+\frac{\epsilon}{2} \frac{T+1}{T-1} h_{x x}-\frac{\epsilon}{2} v_{x x}=0, \quad v_{t}+v v_{x}+\frac{\epsilon}{2} \frac{T+1}{T-1} h_{x x}-\frac{\epsilon}{2} u_{x x}=0,
$$

(we refer to [2] for similar integrable systems of ILW type). System (42) possesses the Lax pair which can be obtained from Lax pair (28) by substituting $\psi \rightarrow \psi e^{k x}$ and then setting $y=-x$ :

$$
\left(\epsilon \partial_{x}+v\right) T \psi=\lambda\left(\epsilon \partial_{x}+u\right) \psi, \quad \epsilon \psi_{t}=\frac{\epsilon^{2}}{2} \psi_{x x}+\left(w-\frac{\epsilon}{2} h_{x}\right) \psi
$$

where $w=\frac{\epsilon}{2} \frac{T+1}{T-1} h_{x}$ and $\lambda=e^{-\epsilon k}$ is a spectral parameter. Truncating the expansion

$$
\frac{\epsilon}{2} \frac{T+1}{T-1}=\frac{\epsilon}{2} \frac{e^{\epsilon \partial_{x}}+1}{e^{\epsilon \partial_{x}}-1}=\frac{\epsilon}{2} \operatorname{coth}\left(\frac{\epsilon}{2} \partial_{x}\right)=\partial_{x}^{-1}+\frac{\epsilon^{2}}{12} \partial_{x}-\frac{\epsilon^{4}}{720} \partial_{x}^{3}+\ldots,
$$

one can obtain nearly integrable dispersive approximations to system (42).

Analogous integrable dispersive regularisations can be constructed for multilayer shear flows with coinciding vorticities within each layer (such flows can model several submerged parallel jets). As an example let us consider the two-layer case (setting again $\omega=g=1$ ):

$$
u_{t}^{i}+u^{i} u_{x}^{i}+h_{x}=0, \quad v_{t}^{i}+v^{i} v_{x}^{i}+h_{x}=0, \quad h=\sum\left(v^{k}-u^{k}\right),
$$

$i=1,2$. This system possesses integrable dispersive regularisation

$$
\begin{aligned}
& u_{t}^{1}+u^{1} u_{x}^{1}+\frac{\epsilon}{2} \frac{T+1}{T-1} h_{x x}-\frac{\epsilon}{2}\left(v^{1}+v^{2}+u^{2}\right)_{x x}=0 \\
& u_{t}^{2}+u^{2} u_{x}^{2}+\frac{\epsilon}{2} \frac{T+1}{T-1} h_{x x}-\frac{\epsilon}{2}\left(v^{1}+v^{2}-u^{1}\right)_{x x}=0 \\
& v_{t}^{1}+v^{1} v_{x}^{1}+\frac{\epsilon}{2} \frac{T+1}{T-1} h_{x x}-\frac{\epsilon}{2}\left(u^{1}+u^{2}+v^{2}\right)_{x x}=0, \\
& v_{t}^{2}+v^{2} v_{x}^{2}+\frac{\epsilon}{2} \frac{T+1}{T-1} h_{x x}-\frac{\epsilon}{2}\left(u^{1}+u^{2}-v^{1}\right)_{x x}=0,
\end{aligned}
$$

which results from the second-order dispersive deformation constructed in Section 3.6 by setting $y=-x$. The corresponding Lax pair is

$$
\left(\epsilon \partial_{x}+v^{1}\right)\left(\epsilon \partial_{x}+v^{2}\right) T \psi=\lambda\left(\epsilon \partial_{x}+u^{1}\right)\left(\epsilon \partial_{x}+u^{2}\right) \psi, \quad \epsilon \psi_{t}=\frac{\epsilon^{2}}{2} \psi_{x x}+\left(w-\frac{\epsilon}{2} h_{x}\right) \psi
$$

where $w=\frac{\epsilon}{2} \frac{T+1}{T-1} h_{x}$ and $\lambda$ is a spectral parameter.

The general $n$-layer case possesses integrable dispersive regularisation

$$
\begin{aligned}
& u_{t}^{i}+u^{i} u_{x}^{i}+\frac{\epsilon}{2} \frac{T+1}{T-1} h_{x x}-\frac{\epsilon}{2}\left(v^{1}+\cdots+v^{n}\right)_{x x}-\frac{\epsilon}{2} D_{j}^{i} u_{x x}^{j}=0, \\
& v_{t}^{i}+v^{i} v_{x}^{i}+\frac{\epsilon}{2} \frac{T+1}{T-1} h_{x x}-\frac{\epsilon}{2}\left(u^{1}+\cdots+u^{n}\right)_{x x}-\frac{\epsilon}{2} D_{j}^{i} v_{x x}^{j}=0
\end{aligned}
$$

where $h=\sum\left(v^{k}-u^{k}\right)$ and $D_{j}^{i}$ is the $n \times n$ skew-symmetric matrix as in Section 3.6. The corresponding Lax pair is

where $w=\frac{\epsilon}{2} \frac{T+1}{T-1} h_{x}$ and $\lambda$ is a spectral parameter.

$$
\prod\left(\epsilon \partial_{x}+v^{k}\right) T \psi=\lambda \prod\left(\epsilon \partial_{x}+u^{k}\right) \psi, \quad \epsilon \psi_{t}=\frac{\epsilon^{2}}{2} \psi_{x x}+\left(w-\frac{\epsilon}{2} h_{x}\right) \psi
$$




\section{Concluding remarks}

Here we list some problems for further study.

- It would be interesting to extend our classification to higher-order analogues of systems (8). As an example let us consider the following 1-parameter deformation of the Lax pair (14):

$$
\begin{aligned}
& T \psi=\frac{\epsilon^{2}}{4} \psi_{y y}-\epsilon u \psi_{y}+\left(u^{2}+\epsilon \lambda u_{y}-2 v\right) \psi, \\
& \epsilon \psi_{t}=\frac{\epsilon^{2}}{4} \psi_{y y}+\left(2 w-\epsilon u_{y}\right) \psi .
\end{aligned}
$$

The compatibility conditions give the system

$$
\begin{aligned}
& u_{t}=u u_{y}+v_{y}+w_{y}-\epsilon \frac{2 \lambda+1}{4} u_{y y} \\
& v_{t}=2 v u_{y}+u v_{y}+\epsilon \frac{2 \lambda+1}{4}\left(v_{y y}+w_{y y}-u_{y}^{2}\right)-\epsilon^{2} \frac{2 \lambda^{2}+2 \lambda+1}{8} u_{y y y},
\end{aligned}
$$

with the nonlocality $\triangle w=\frac{T+1}{2} u_{y}$. Note that the third-order derivative $u_{y y y}$ disappears only for the particular parameter values $\lambda=\frac{-1 \pm i}{2}$, which leads to system (13). Under the differential substitution $\tilde{v}=v-\epsilon \frac{2 \lambda+1}{4} u_{y}$, equation (44) takes $\lambda$-independent form,

$$
\begin{aligned}
& u_{t}=u u_{y}+\tilde{v}_{y}+w_{y}, \\
& \tilde{v}_{t}=2 \tilde{v} u_{y}+u \tilde{v}_{y}-\frac{\epsilon^{2}}{16} u_{y y y} .
\end{aligned}
$$

Thus, there exist nontrivial higher-order integrable systems with nonlocality of ILW type. It would be of interest to classify third-order integrable extensions of systems (8).

- Looking for integrable dispersive deformations of various dispersionless integrable systems, we came across the following (unforeseen) phenomenon: there exists (numerous) examples of 3D dispersionless integrable systems $\Sigma$ possessing integrable dispersive deformations which, however, are not compatible with reductions of $\Sigma$. As an illustration let us consider the dispersionless integrable system from Section 3.5,

$$
u_{t}^{i}=u^{i} u_{y}^{i}+w_{y}, \quad w_{x}=\left(u^{1}+u^{2}+u^{3}\right)_{y},
$$

$i=1,2,3$, which possesses integrable dispersive deformation

$$
\begin{aligned}
u_{t}^{1} & =u^{1} u_{y}^{1}+w_{y}+\frac{\epsilon}{2}\left(u^{2}+u^{3}\right)_{y y}, \\
u_{t}^{2} & =u^{2} u_{y}^{2}+w_{y}+\frac{\epsilon}{2}\left(-u^{1}+u^{3}\right)_{y y}, \\
u_{t}^{3} & =u^{3} u_{y}^{3}+w_{y}+\frac{\epsilon}{2}\left(-u^{1}-u^{2}\right)_{y y}, \\
\triangle w & =\frac{T+1}{2}\left(u^{1}+u^{2}+u^{3}\right)_{y} .
\end{aligned}
$$

At the dispersionless level, this system possesses a reduction $u^{3}=u^{2}$,

$$
u_{t}^{1}=u^{1} u_{y}^{1}+w_{y}, \quad u_{t}^{2}=u^{2} u_{y}^{2}+w_{y}, \quad w_{x}=\left(u^{1}+2 u^{2}\right)_{y},
$$

which, however, is not compatible with the dispersive deformation. Attempts to seek integrable dispersive deformations of system (45) in the form

$$
\begin{aligned}
u_{t}^{1} & =u^{1} u_{y}^{1}+w_{y}+\epsilon(\ldots)+\epsilon^{2}(\ldots)+\ldots, \\
u_{t}^{2} & =u^{2} u_{y}^{2}+w_{y}+\epsilon(\ldots)+\epsilon^{2}(\ldots)+\ldots, \\
\triangle w & =\frac{T+1}{2}\left(u^{1}+2 u^{2}\right)_{y}
\end{aligned}
$$

where dots at $\epsilon^{n}$ denote differential polynomials of degree $n+1$ in the $y$-derivatives of $u^{1}, u^{2}$ and $w$, lead to a contradiction at the order $\epsilon^{2}$.

It is a challenging problem to find out whether the $2+1$ dimensional waterbag system (24) possesses integrable dispersive deformations for rational values of the parameters $\omega_{k}$. This would provide a dispersive regularisation of system (38) governing propagation of long nonlinear waves in a shear flow with piecewise linear velocity profile (and rational vorticities). 
- Integrable dispersive regularisations of system (40) constructed in this paper provide a framework which could be used to investigate the evolution of dispersive shock waves arising from suitable initial conditions.

\section{Acknowledgments}

We thank A. Chesnokov, S. Gavrilyuk, K. Khusnutdinova and A. Pogrebkov for useful discussions. The research of EVF was supported by a grant from the Russian Science Foundation No. 21-11-00006, https://rscf.ru/project/21-11-00006/. The research of VSN was supported by the EPSRC grant EP/V050451/1.

\section{References}

[1] L.V. Bogdanov, B.G. Konopelchenko, Symmetry constraints for dispersionless integrable equations and systems of hydrodynamic type, Phys. Lett. A 330, no. 6 (2004) 448-459.

[2] B.K. Berntson, E. Langmann, J. Lenells, On the non-chiral intermediate long wave equation, arXiv:2005.10781,

[3] A.A. Chesnokov, G.A. El, S.L. Gavrilyuk, M.V. Pavlov, Stability of shear shallow water flows with free surface, SIAM J. Appl. Math. 77, no. 3 (2017) 1068-1087.

[4] E. Date, M. Jimbo and T. Miwa, J. Phys. Soc. Japan 51 (1982) 4116.

[5] B.A. Dubrovin and Youjin Zhang, Bi-Hamiltonian hierarchies in 2D topological field theory at one-loop approximation, Comm. Math. Phys. 198 (1998) no. 2, 311-361.

[6] B. Dubrovin, S-Q. Liu, Y. Zhang, On Hamiltonian perturbations of hyperbolic systems of conservation laws. I. Quasi-triviality of bi-Hamiltonian perturbations. Comm. Pure Appl. Math. 59, no. 4 (2006) 559-615.

[7] E.V. Ferapontov and K.R. Khusnutdinova, On integrability of (2+1)-dimensional quasilinear systems, Comm. Math. Phys. 248 (2004) 187-206.

[8] E.V. Ferapontov and A. Moro, Dispersive deformations of hydrodynamic reductions of 2D dispersionless integrable systems, J. Phys. A: Math. Theor. 42 (2009) 035211, 15pp.

[9] E.V. Ferapontov, A. Moro and V.S. Novikov, Integrable equations in $2+1$ dimensions: deformations of dispersionless limits, J. Phys. A: Math. Theor. 42 (2009) (18pp).

[10] E.V. Ferapontov, K.R. Khusnutdinova, The Haantjes tensor and double waves for multi-dimensional systems of hydrodynamic type: a necessary condition for integrability Proc. R. Soc. A 462 (2006) 1197-1219.

[11] E.V. Ferapontov, V.S. Novikov and I. Roustemoglou, Towards the classification of integrable differentialdifference equations in $2+1$ dimensions, J. Phys. A: Math. Theor. 46 (2013) 13pp.

[12] E.V. Ferapontov, V. Novikov and I. Roustemoglou, On the classification of discrete Hirota-type equations in 3D, IMRN no. 13 (2015) 4933-4974; doi: 10.1093/imrn/rnu086.

[13] J. Gibbons and S.P. Tsarev, Reductions of the Benney equations, Phys. Lett. A 211 (1996) 19-24.

[14] J. Gibbons and S.P. Tsarev, Conformal maps and reductions of the Benney equations, Phys. Lett. A 258 (1999) 263-271.

[15] J. Haantjes, On $X_{m}$-forming sets of eigenvectors, Indagationes Mathematicae 17 (1955) 158-162.

[16] B. Huard and V.S. Novikov, Classification of integrable Davey-Stewartson type equations, J. Phys. A 46, no. 27 (2013) 275202, 13 pp.

[17] V.S. Novikov and E.V. Ferapontov, On the classification of scalar evolutionary integrable equations in $2+1$ dimensions, J. Math. Phys. 52, 2 (2011) 023516; DOI: 10.1063/1.3554695.

[18] Xian-min Qian, Sen-yue Lou and Xing-biao Hu, Variable Separation Approach for a Differential-difference Asymmetric Nizhnik-Novikov-Veselov Equation, Z. Naturforsch. 59a (2004), 645-658. 
[19] T. Tamizhmani, V.S. Kanaga and K.M. Tamizhmani, Wronskian and rational solutions of the differentialdifference KP equation, J. Phys. A 31 (1998) 7627-7633.

[20] V.E. Zakharov, Dispersionless limit of integrable systems in $2+1$ dimensions, in Singular Limits of Dispersive Waves, Ed. N.M. Ercolani et al., Plenum Press, NY (1994) 165-174.

[21] V.E. Zakharov, A.V. Odesskii, M. Cisternino, M. Onorato, Five-wave classical scattering matrix and integrable equations, Theoret. and Math. Phys. 180, no. 1 (2014) 759-764.

[22] V. E. Zakharov, Benney equations and quasi-classical approximation in the method of inverse problem, Funk. Anal. Prilozh., 14 (1980) 15-24. 